\title{
Implementation of a Rapid Post-Code Debrief Quality Improvement Project in a Community Emergency Department Setting
}

\author{
Tomasz Przednowek, DO' ${ }^{\circ}$, Camille Stacey, RN, MSN', Katherine Baird, BS, RRT, RRT-ACCS², Robert Nolan, DO', Jesse \\ Kellar, MD, MBA ${ }^{3}$, William D. Corser, PhD, RN, NEA-BC ${ }^{4}$ \\ ${ }^{1}$ Emergency Medicine, Spectrum Health Lakeland, ${ }^{2}$ Respiratory Therapy, Spectrum Health Lakeland, ${ }^{3}$ Emergency Medicine, Saint Agnes Medical \\ Center, ${ }^{4}$ Statewide Campus System, Michigan State University College of Osteopathic Medicine \\ Keywords: emergency medicine, code, debriefing, quality improvement, post-code debriefing, nursing, emotional support, wellness, survey, trauma \\ https://doi.org/10.51894/001c.21376
}

\section{Spartan Medical Research Journal}

Vol. 6, Issue 1, 2021

\section{CONTEXT}

Regular debriefing has been associated with improved resource utilization and measurable improvements in team performance in crisis situations. While Emergency Department (ED) staff have often stated that they would like to be provided a formal debriefing model after "code blue" and similar events, few EDs have such protocols in place.

\section{METHODS}

The study consisted of two data collection processes: (1) completion of a 7-item survey distributed pre-intervention, 6-months post-intervention, and 1-year post-intervention, and (2) completion of a Rapid Post-Code Debriefing form. Overall responses were measured on a possible 0-10 scale and individual responses were tracked. The debrief process was triggered by one of four criteria and followed a standard format using a readily available form.

\section{RESULTS}

A total of 178 pre- and post-debriefing protocol implementation survey responses were collected throughout the duration of the study. Of those, 79 (44.4\%) were pre-protocol response surveys. The post-protocol responses were comprised of 51 (51.5\%) six month and 48 (48.5\%) 12 -month surveys. The average overall satisfaction with code-response performance increased significantly following the implementation of the debriefing protocol, from $\mathrm{M}=6.661, \mathrm{SD}=2.028$ to $\mathrm{M}=7.90, \mathrm{SD}=1.359$ (independent $\mathrm{t}$-test $=5.069$, $\mathrm{p}<0.001)$. There was a statistically significant decrease regarding how respondents felt emotionally supported after a code by their staff, (Pearson Chi Square 14.977, df 4, p = 0.005).

\section{CONCLUSION}

During this study, implementation of a post-code debriefing resulted in increased overall satisfaction with how codes had been conducted and there was a significant change in how staff felt in regards to code team leaders and an expectation of "returning to work." However, there a noted overall decrease in perceptions of feeling supported by other staff involved during the code. Further studies in both community and academic-based ED settings are needed to further explore these complex relationships.

\section{INTRODUCTION}

\section{BACKGROUND}

Healthcare workers in emergency department (ED) settings are at routine risk of being exposed to potentially traumatic events and these patient care situations often have a profound impact on the staff involved. Debriefing, which allows for emotional processing and reflection upon areas for possible improvement, has been found to be one way in which to increase overall performance, reduce equipment-related problems, and improve communication and teamwork. ${ }^{1,2}$

However, despite being well established in the military and other high-stakes industries such as aviation, debriefing remains poorly established in the ED settings. ${ }^{3,4}$ Given that the benefits of post-simulation debriefings have been widely accepted in other settings, there is likely significant utility in implementing a debriefing protocol in the Emergency Department as well. ${ }^{2,5,6}$ 


\section{IMPORTANCE}

While staff have often stated that they would like to be provided a formal debriefing model after treating a cardiac arrest or other critically ill patient, few EDs have such protocols in place and there is often no formal training offered during medical education on how to debrief after a situation. ${ }^{4,7-10}$ When given the option, however, people usually prefer to debrief with persons facing the same stressful situation. ${ }^{11}$

When healthcare clinicians in these types of situations are allowed to debrief, it leads to increased empathy, normalization, and validation, which can have significant stress moderating effects. ${ }^{5,12,13}$ Regular debriefing has been associated with improved resource utilization and measurable improvements in team performance in crisis situations. 1,2,5,14-16 Implementing a standardized debriefing process encourages a supportive team-based culture, improves the transition to patient care activities after the event, increases feelings of support by peers and leaders, and improves time to regroup prior to returning to work assignments. ${ }^{12,17}$

\section{GOALS OF THIS INVESTIGATION}

The goals of this quality improvement project were to assess: a) the overall baseline satisfaction with how resuscitations had been performed, b) perceptions of any confusion during resuscitations, problems with lack of equipment/ medications/staffing, c) level of perceived emotional support after codes, and d) reoccurrence of associated thoughts with a given code over 24 hours. The overall hypotheses were that a) baseline satisfaction with how resuscitations had been performed would improve, b) confusion during resuscitations and missing equipment/medications/staffing would decrease, $c$ ) the level of perceived emotional support after a code would improve and d) there would be a decreased reoccurrence of associated thoughts with a given code over 24 hours.

\section{METHODS}

Before data collection, the study was determined exempt by the authors' institutional review board in June 2018. The study consisted of a survey portion and a Rapid Post-Code Debrief form (see supplement) which were collected at two community-based Emergency Departments. The Lakeland seven-item survey was created with this goal in mind, in part based on a previous, non-externally validated survey from a previous study in an ED setting and partially created de-novo with staff input from physicians, nurses, physical therapists, and emergency technicians from the EDs participating in this study. ${ }^{17}$ The brief 7 -item survey was distributed three times: prior to implementation of the Rapid Post-Code Debriefing, after 6 months of debriefings, and at the one-year mark post implementation.

Overall responses were measured on a possible $0-10$ scale ("0" meaning "completely unsatisfied/never" and " 10 " meaning "completely satisfied/often") and individual responses were tracked using an anonymous, unique ID created by each person. Each participant was also asked to identify their role on the healthcare team, and overall trends were assessed regarding responses to each of the survey items. The survey was hand distributed during morning staff meetings to each staff member working in the Emergency Department who participated in the debriefing process. These included nurses, patient care technicians, respiratory therapists, attending physicians, and resident physicians. In addition to polling during morning staff meetings, staff were also polled at various times of the day, as well as in different locations, and on different days of the week.

Survey responses, while identifiable by a unique code created by each participant, could not be linked by the researcher to any specific individual at any time since the exact code used by each person was only known by the person generating the code and they were collected in anonymous envelopes.

The debrief process was triggered by one of four criteria (see "Rapid Post-Code Debrief" form below). Forms were available at the clerk's desk. Although the Rapid Post-Code Debrief form was most often filled out by the charge nurse or documenting nurse immediately following a qualifying event, anyone was able to initiate the process and anyone was allowed to self-exclude for any reason, such as when it was felt debriefing was a lower priority relative to other operating needs at the time (e.g., other urgent staff/patient needs). Individual names were not recorded of those in attendance, nor was this process used in any way punitively against staff who chose to participate. While there was a check box on the form regarding staff occupation, no additional demographic information (e.g., age, gender, tenure on ED staff, etc.) was collected or analyzed in this study.

Instructions on how the debrief was performed, what triggered a debriefing, and information collected on the debrief form can be seen below.

\section{PRIMARY DATA ANALYSIS}

After data collection was completed, the surveys were analyzed using a series of independent-sample t-tests and Chi Square crosstabulations and graphs. ${ }^{18}$ Due to the difficulty in tracking individual responses across the three time intervals to survey questions, all responses were categorized into thirds. Those most unhappy with lowest scores were placed into one group, there was a second group for those with a medium score, and finally those with the highest scores were in a final third group. Changes were then measured across the three groups across the study period (e.g., asking "did the bottom third of scores improve over time?"). Also, given difficulty with obtaining survey responses, many analyses (unless otherwise specifically stated) combined the six-month responses with the one-year responses as simply a "post implementation group". A series of non-parametric stepwise multinomial regression analytic procedures were performed to examine for other potential factors influencing responses. ${ }^{19}$ All analytic procedures were conducted using S.P.S.S. version 25 analytic software observing a twotailed coefficient Alpha p value of 0.05 to indicate statistical significance. $^{20}$ 


\section{Lakeland Health Rapid Post-Code Debrief}

Date:

Time Started:

Time Completed:

\section{Patient Outcome}

$\square$ Alive

$\square$ Expired

\section{Location:}

$\square$ Emergency Department

$\square$ Critical Care Unit

$\square$ Progressive Care Unit

$\square$ Ortho/Neuro

$\square$ Post-Surgical

$\square$ Cardiac Telemetry

$\square$ Medical-Oncology

\section{If Debrief Not Done:}

$\square$ Too Many Urgent Patients

$\square$ Did not Feel it was Needed

$\square$ Other:

\section{Staff Present:}

$\square$ Charge Nurse

$\square$ Documenting Nurse

$\square$ Code Leader

$\square$ Attending Physician

$\square$ Resident Physician

$\square$ Respiratory Therapist

$\square$ Tech/Nurse Assistant

$\square$

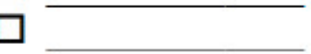

\section{Interventions Done:}

$\square$ CPR

$\square$ Intubation

$\square$ Trauma Level I

Called

$\square$ Rapid Response

Called

$\square$ Defibrillation

Reason Debrief

Called:

$\square$ Cardiac Arrest

(Pulseless)

$\square$ Medical (including seizure, Code Stroke)

$\square$ Respiratory Arrest

$\square$ Trauma Level I

$\square$ Pediatric Patient

What went well during our care of the patient?

What could have gone better during the care of our patient?

Is there anything anyone needs prior to going back to work?

Was anyone confused at any time during the resuscitation who the team leader was?
Was the Physician Team Leader the only doctor calling out orders?

\section{Yes $\square$ No}

\section{Yes $\square$ No}

Advice for the debriefing: - Please start by thanking members for being present and encouraging everyone to participate. If patient did not survive after the resuscitation, please take a moment of silence prior to starting the debrief to honor and recognize the patient. Then, please state at the beginning: "The purpose of this debriefing is for education, quality improvement, and emotional processing. It is not for blaming or penalization, and names are not recorded during this session. This meeting will take five to ten minutes. If you have urgent issues to attend to, you are welcome to leave at any time. "After the introduction, please summarize the patient's course (in less than $1 \mathrm{~min}$ ) and then proceed to the group discussion. 
Table 1 - Statistical Results of each Survey Item

\begin{tabular}{|l|l|}
\hline Survey item & Results of Pearson Chi Square test* \\
\hline Overall satisfaction & $\begin{array}{l}\text { Pearson Chi Square }=37.377, \mathrm{df} 10, \mathrm{p}<0.001 \text { (continuous } \\
\text { measure), } \\
\text { Pearson Chi Square }=16.561, \mathrm{df} 2, \mathrm{p}<0.001 \text { (categorical } \\
\text { measure) }\end{array}$ \\
\hline Number of people running a code & Pearson Chi Square $=10.945 . \mathrm{df} 4, \mathrm{p}=0.027$ \\
\hline Frequency of items missing & Pearson Chi Square $=2.648, \mathrm{df} 4, \mathrm{p}=0.618$ \\
\hline $\begin{array}{l}\text { Appropriate number of staff present in the room did not } \\
\text { change }\end{array}$ & Pearson Chi Square $=8.428, \mathrm{df} 4, \mathrm{p}=0.077$ \\
\hline Thoughts about just returning to work & Pearson Chi Square 11.351, df 4, $\mathrm{p}=0.023$ \\
\hline Recurring thoughts about the code & Pearson Chi Square 4.644, df 4, $\mathrm{p}=0.324$ \\
\hline Felt emotionally supported after a code by their staff & Pearson Chi Square 14.977, df 4, $\mathrm{p}=0.005$ \\
\hline
\end{tabular}

* Statistically Significant Differences appear in Bold Font

\section{RESULTS}

A total of 178 pre- and post-debriefing protocol implementation survey responses were collected throughout the duration of the study. Of those, 79 (44.4\%) were pre-protocol response surveys. The post-protocol responses were comprised of 51 (51.5\%) six month and 48 (48.5\%) 12-month surveys. Of the 138 respondents who reported their professional role, 30 (21.7\% of reported) were physicians, 56 (40.6\%) were nurses and 52 (37.7\%) were other types of healthcare personnel. Approximately 40 (22.5\%) staff members chose not to disclose their professional roles. Every attempt was made to match participants at each of the survey intervals based on the respondent's unique identifier, although ultimately the majority of surveys could not be matched due to either missing identifiers or the respondents not remembering their identifier.

Notably, the average overall code satisfaction pre-protocol (Mean $=6.661$, SD 2.028) was significantly lower than the average of combined six and year post-protocol survey responses, or "post implementation" (Mean $=7.90, \mathrm{SD}$ 1.359) (independent t-test $=5.069, \mathrm{p}<0.001$ ). The average post-implementation satisfaction of respondents was 7.33 (SD 1.80) and ranged from 0 ("Not at All Satisfied") through 10 ("Completely Satisfied"). Each survey had a total possible score calculated by adding responses to each question (with a maximum score of 60), and scores ranged from 13 through 56 on pre-implementation surveys and on the surveys at six months and one year post implementation (mean 36.89 (SD 7.30)).

Overall code satisfaction ratings increased significantly after implementation of the debriefing (Pearson Chi Square $=37.377$, df 10, $\mathrm{p}<0.001$ (continuous measure), Pearson Chi Square $=16.561$, df 2, p $<0.001$ (categorical measure) (Table 1, Figure 1, Figure 2). Different results were observed when responses were stratified by provider type (Pearson Chi Square $=13.271$, df 8, p=0.103). Only two other survey questions showed improvement over the study period (e.g., "Number of people running a code," and "Thoughts about returning to work") (Table 1 ). Finally, there was a statistically significant decrease regarding how respondents felt emotionally supported after a code by their staff, (Pearson Chi Square 14.977, df 4, p = 0.005) (Table 1, Figure 3).

Finally, a series of multinomial predictive models was performed to look at the significance of each selected study measures on overall code satisfaction, and only survey time (pre-protocol vs post-protocol period), increased satisfaction significantly (Likelihood Ratio Chi Square $=16.671$, df $4, \mathrm{p}=0.002)$.

\section{DISCUSSION}

These main findings indicate that implementation of a post-code debriefing can positively impact overall satisfaction of how a code is run. In regard to the other areas assessed by the survey there were two other statistically significant changes, including a measured lower perception of emotional support after debriefing protocol implementation.

Much like in the Copeland et al. study which also implemented debriefings in the Emergency Department, the staff involved in this study also had a statistically significant improvement in overall satisfaction. ${ }^{17}$ Indeed, the overall response to implementation of this protocol was positive, and while it not directly measured on the survey, staff verbally indicated during morning meetings that they liked being able to debrief with persons who faced the same stressful situation, similar to what has been seen previously in the literature. ${ }^{11}$ The number of people running a code and thoughts about returning to work were also improved in a statistically significant manner, which is in line with previous findings such as those in the Copeland et al. study. ${ }^{17}$

The finding that staff felt less overall supported by other staff members after implementing the debriefing protocol is unexpected and goes against what was found in the similar previous study by Copeland et al. ${ }^{17}$ Perhaps this is due to the staff paying more critical attention to this aspect of a debriefing after code events and their work environment, or perhaps there may be other factors involved. Further studies may help elucidate the exact etiology behind this finding. 


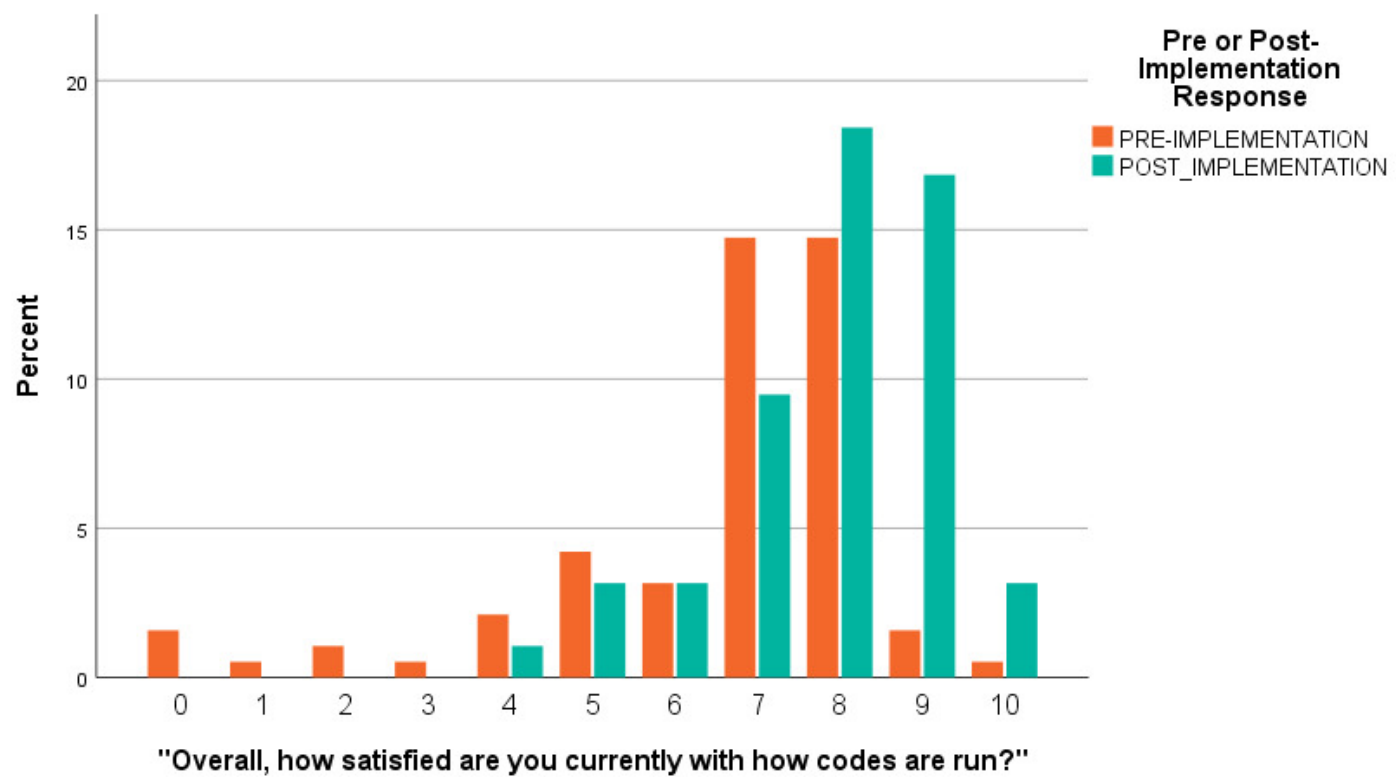

Figure 1 - Continuous Overall Satisfaction Pre-implementation vs Post-Implementation

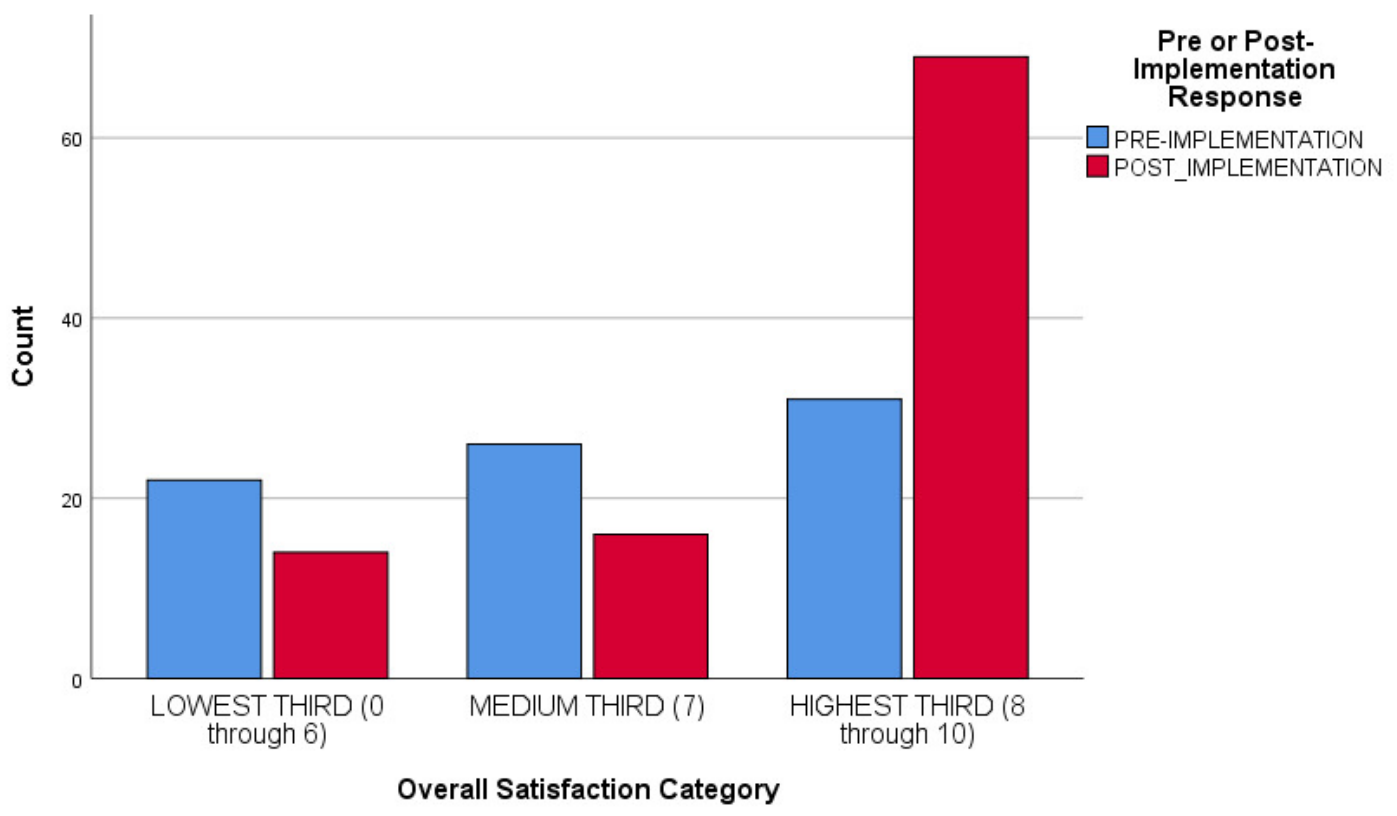

Figure 2 - Overall Satisfaction, Pre- and Post-Implementation

\section{LIMITATIONS}

The study has several limitations. Some of these non-significant results could be attributed to lack of a sufficientsized and/or diverse enough sample. The sample size included surveys which did not have a unique identifier which could be tracked throughout the study period, either due to the participants not including one on the form altogether or forgetting their unique ID during the three survey periods and inventing a new one each time. Results may also have been skewed by "preferred response" pressures in which some respondents may have felt pressure to indicate that the protocol helped them after code events. Additionally, these findings may not possess "external generalizability" to non-community-based ED settings. 


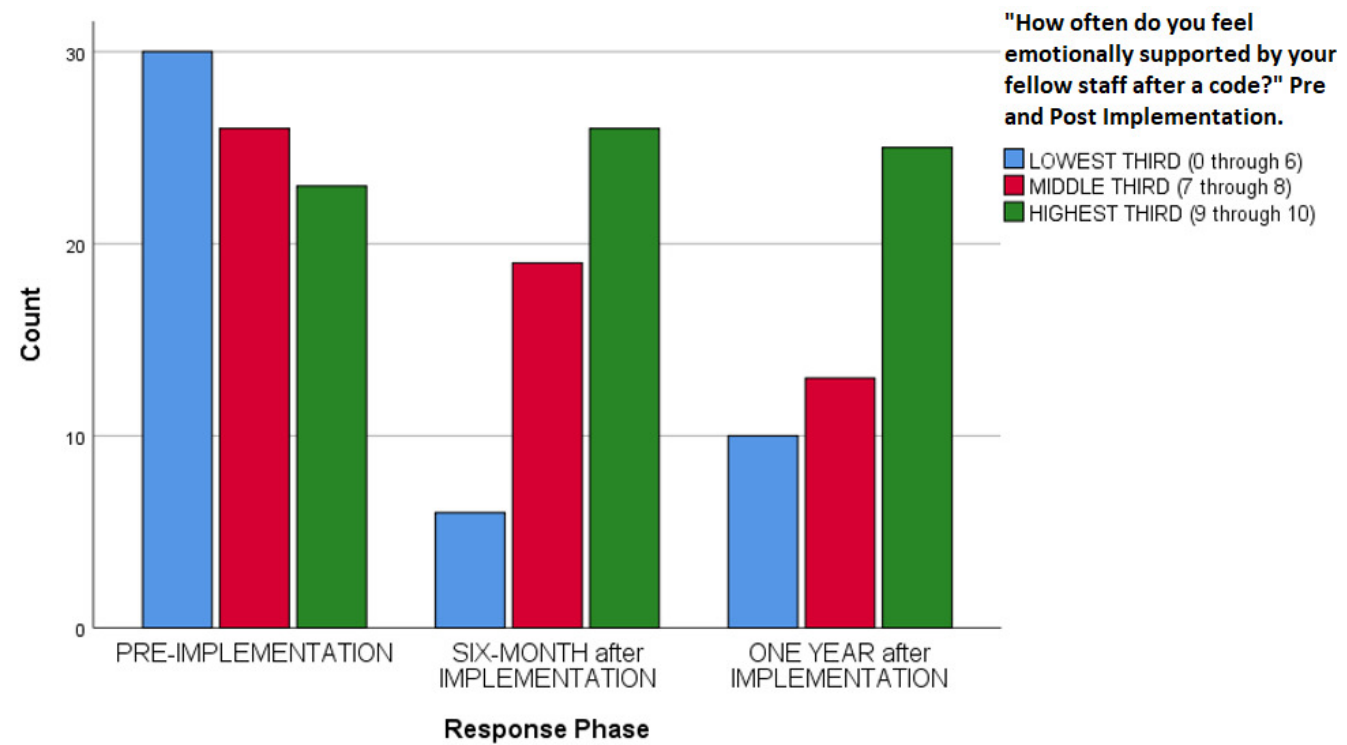

Figure 3 - Perceive Emotional Support

Although every effort was made to maximize the survey response rate, it was not possible to ensure all eligible staff filled out a survey, nor could it be ensured that staff filled out surveys at all three time points. Every attempt was made to poll staff at different times of the day, in different locations, and on different days of the week. However, despite this, absolute survey response rates declined at each time interval (going from 78 (pre-implementation) responses to 51 (six-month) and then 48 (12-month) responses).

There were several debriefing-related variables which we could not control for, such as who led each debriefing, the exact timing of when the debrief was delivered, and so on. There was also no way to control when the actual events which trigger a debriefing would occur, nor was it possible to estimate what portion of critical events went without a debriefing, especially early in the project.

Cultural, gender, social, and educational background differences often play a role in how people process information and cope with acute events and can limit the amount of support a debriefing can provide. ${ }^{21,22}$ Furthermore, certain skills, such as high-quality CPR, may simply already be done well enough that further quality improvement processes may not have been of much benefit, thus impacting the maximum possible satisfaction scores. ${ }^{23}$

\section{CONCLUSION}

During this study, implementation of a post-code debriefing resulted in increased overall satisfaction with how codes had been conducted. There was also a significant change in how staff felt in regard to code team leaders and an expectation of "returning to work." However, there a noted overall decrease in perceptions of feeling supported by other staff involved during the code. Further studies in both community and academic-based ED settings are certainly needed to further explore these complex relationships.

Submitted: October 28, 2020 EDT, Accepted: February 26, 2021 EDT 


\section{REFERENCES}

1. Boet S, Bould MD, Sharma B, et al. Within-team debriefing versus instructor-led debriefing for simulation-based education: A randomized controlled trial. Ann Surg. 2013;258(1):53-58. doi:10.1 $\underline{097 / \text { sla.0b013e31829659e4 }}$

2. Eppich WJ, Mullan PC, Brett-Fleegler M, Cheng A. "Let's Talk About It": Translating Lessons From Health Care Simulation to Clinical Event Debriefings and Coaching Conversations. Clinical Pediatric Emergency Medicine. 2016;17(3):200-211. doi:10.1016/ j.cpem.2016.07.001

3. Kaplan Z, Iancu I, Bodner E. A review of psychological debriefing after extreme stress. Psychiatr Serv. 2001;52(6):824-827. doi:10.1176/appi.p $\underline{\text { s.52.6.824 }}$

4. Nocera M, Merritt C, Santen S. Pediatric Critical Event Debriefing in Emergency Medicine Training: An Opportunity for Educational Improvement. AEM Education and Training. 2017;1(3):208-214. doi:10.100 2/aet2.10031

5. Gardner R. Introduction to debriefing. Semin Perinatol. 2013;37(3):166-174. doi:10.1053/j.semper i.2013.02.008

6. Kessler DO, Cheng A, Mullan PC. Debriefing in the emergency department after clinical events: A practical guide. Ann Emerg Med. 2015;65(6):690-698. doi:10.1016/j.annemergmed.2014.10.019

7. Healy S, Tyrrell M. Importance of debriefing following critical incidents. Emerg Nurse. 2013;20(10):32-37. doi:10.7748/en2013.03.20.10.32.s8

8. Nadir N-A, Bentley S, Papanagnou D, Bajaj K, Rinnert S, Sinert R. Characteristics of Real-Time, Non-Critical Incident Debriefing Practices in the Emergency Department. West J Emerg Med. 2017;18(1):146-151. doi:10.5811/westjem.2016.10.31 $\underline{467}$

9. Theophilos T, Magyar J, Babl FE. Debriefing critical incidents in the paediatric emergency department: Current practice and perceived needs in Australia and New Zealand. Emerg Med Australas. 2009;21(6):479-483. doi:10.1111/j.1742-6723.2009.01 231.x

10. Zinns LE, O’Connell KJ, Mullan PC, Ryan LM, Wratney AT. National Survey of Pediatric Emergency Medicine Fellows on Debriefing After Medical Resuscitations. Pediatr Emerg Care. 2015;31(8):551-554. doi:10.1097/pec.0000000000000 196
11. Calder-Sprackman SM, Kumar T, Sampsel K, Gerin-Lajoie C. LO42: Ice Cream Rounds: The adaptation and implementation of a peer-support wellness rounds in an emergency medicine residency training program. CJEM. 2017;19(S1):S42. doi:10.101 $\underline{\text { 7/cem.2017.104 }}$

12. Jenkins SR. Social Support and Debriefing Efficacy Among Emergency Medical Workers After a Mass Shooting Incident. $R$ Journal of Social Behavior and Personality. 1996;11(3):477. doi:10.1007/s12144-997-1 $\underline{012-1}$

13. Ruzek JI. Providing "brief education and support" for emergency response workers: An alternative to debriefing. Military Medicine. 2002;167(9):73-75. doi:1 $\underline{0.1093 / \mathrm{milmed} / 167 . \mathrm{suppl} 4.73}$

14. Dyregrov A. The Process in Psychological Debriefings. J Traum Stress. 1997;10(4):589-605. doi:1 $\underline{0.1002 / i t s .2490100406}$

15. Mullan PC, Cochrane NH, Chamberlain JM, et al. Accuracy of Postresuscitation Team Debriefings in a Pediatric Emergency Department. Ann Emerg Med. 2017;70(3):311-319. doi:10.1016/j.annemergmed.201 $\underline{7.01 .034}$

16. Sawyer T, Loren D, Halamek LP. Post-event debriefings during neonatal care: Why are we not doing them, and how can we start? J Perinatol. 2016;36(6):415-419. doi:10.1038/jp.2016.42

17. Copeland D, Liska H. Implementation of a PostCode Pause: Extending Post-Event Debriefing to Include Silence. J Trauma Nurs. 2016;23(2):58-64. do i:10.1097/jtn.0000000000000187

18. Moore DS, McCabe GP. Introduction to the Practice of Statistics. 4th ed. New York, NY: W H Freeman \& Co; 2003.

19. Vittinghoff E, Shiboski SC, Glidden DV, McCulloch CE. Regression Methods in Biostatistics: Linear, Logistic, Survival, and Repeated Measures Models. New York: Brooks/Cole, Springer Science+Business Media, Inc; 2005.

20. IBM Corp. IBM SPSS Statistics for Windows, Version 24.0. 2016.

21. Chung HS, Dieckmann P, Issenberg SB. It is time to consider cultural differences in debriefing. Simul Healthc. 2013;8(3):166-170. doi:10.1097/sih.0b013e31 8291d9ef 
22. Fullerton CS, Ursano RJ, Vance K, et al. Debriefing Following Trauma. Psychiatr Q. 2000;71:259.
23. Couper K, Kimani PK, Davies RP, et al. An evaluation of three methods of in-hospital cardiac arrest educational debriefing: The cardiopulmonary resuscitation debriefing study. Resuscitation.

2016;105:130-137. doi:10.1016/j.resuscitation.2016.0 $\underline{5.005}$ 


\section{Debriefing Survey}

Please take a moment to share your thoughts on how resuscitations (aka "codes") have been conducted in our department over the last 6 months. Please circle your answer to each question below. Your answers are anonymous and will be used to help improve our debriefing process, however, we ask you to use a 4 letter and 2 number ID so we can match individual survey responses before and after implementation of the debriefing and write it in above, as well as circle your current role in the healthca re team. For your identifier please use the first two letters of your favorite food (pizza would be PI), the last two letters of your hometown (Niles would be ES), and the day of your mother's birthday (May 10 would be 10, the full code in this example would be PIES10). Please feel free to include any additional comments on the back page.

Overall, how satisfied are you currently with how codes are run?

$\begin{array}{lllllllllll}0 & 1 & 2 & 3 & 4 & 5 & 6 & 7 & 8 & 9 & 10\end{array}$

Not at all satisfied

Completely satisfied

How often do you feel there is confusion about how many people are running a code?

$\begin{array}{rrrrrrrrrrr}0 & 1 & 2 & 3 & 4 & 5 & 6 & 7 & 8 & 9 & 10 \\ \text { Rarely } & & & & & & & & & & \end{array}$

How often do you feel like something is missing in a code situation (medication, equipment)?

$\begin{array}{rrrrrrrrrrr}0 & 1 & 2 & 3 & 4 & 5 & 6 & 7 & 8 & 9 & 10 \\ \text { Rarely } & & & & & & & & & & \end{array}$

How often do you feel there is an appropriate amount of staff in the room to help?

$\begin{array}{rrrrrrrrrrr}0 & 1 & 2 & 3 & 4 & 5 & 6 & 7 & 8 & 9 & 10 \\ \text { Rarely } & & & & & & & & & \end{array}$

How often do you feel emotionally supported by your fellow staff after a code?

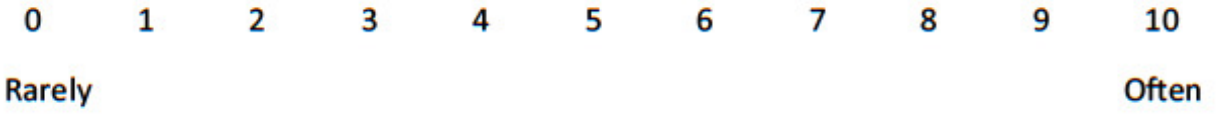

How often do you feel there is an expectation that you will "just return to work" after a code?

$\begin{array}{rrrrrrrrrrr}0 & 1 & 2 & 3 & 4 & 5 & 6 & 7 & 8 & 9 & 10 \\ \text { Rarely } & & & & & & & & & & \end{array}$

How often do you find yourself having recurring thoughts or feelings associated with the code over the course of 24 hours following the event?

$\begin{array}{rrrrrrrrrrr}0 & 1 & 2 & 3 & 4 & 5 & 6 & 7 & 8 & 9 & 10 \\ \text { Rarely } & & & & & & & & & & \end{array}$

